Journal of Mathematics and Statistics 4 (3): 186-193, 2008

ISSN 1549-3644

(C) 2008 Science Publications

\title{
Powerful Features of the Modern Syllogistic Method of Propositional Logic
}

\author{
${ }^{1}$ Ali Muhammad Rushdi and ${ }^{2}$ Omar Mohammed Ba-Rukab \\ ${ }^{1}$ Department of Electrical and Computer Engineering, King Abdulaziz University, \\ P.O. Box 80204, Jeddah 21589, Saudi Arabia \\ ${ }^{2}$ Department of Computer Technology, College of Telecommunication and Electronics, \\ P.O. Box 2816, Jeddah 21461, Saudi Arabia
}

\begin{abstract}
In traditional propositional logic, many replacement and inference rules were involved to ascertain if the truth of several antecedents implied the truth of a particular consequent. This research described a powerful technique called the Modern Syllogistic Method (MSM), which ferreted out from a set of premises all that can be concluded from it and casted the resulting conclusions in the simplest and most compact form. We observed that all replacement rules were explicitly and inherently integrated within the MSM and proved that all inference rules were simply limited special cases of it. This meant that the MSM constituted a complete method of logic deduction. We also showed how to use the MSM in determining whether inconsistencies existed within a given set of premises and also in detecting formal logical fallacies. We demonstrated the applicability of the method in diverse fields via four examples that illustrated its mathematical details and exhibited the nature of conclusions it can come up with. In fact, these examples demonstrated the possibility of extracting deductions that were not so obvious and even surprising. The examples also showed how logic can be misused and how logic misuse can be avoided or detected.
\end{abstract}

Key words: Logic deduction, Modern Syllogistic Method (MSM), completeness, inconsistency, fallacies, logic misuse

\section{INTRODUCTION}

Propositional logic, also called sentential logic, has a long history of more than 2000 years. It can be viewed as a grammar for exploring the construction of complex propositions from atomic statements using logical connectives such as and, or and not. The fundamental inference problem in propositional logic is to ascertain if the truth of several propositions (called antecedents) implies the truth of a particular proposition of interest (called a consequent).

The traditional (symbolic) approach to propositional logic is based on a clear separation of the syntactical and semantical functions. The syntactics deals with the laws that govern the construction of logical formulas from the atomic propositions and with the structure of proof. Semantics, on the other hand, is concerned with the interpretation and meaning associated with the syntactical objects.

Propositional calculus is based on purely syntactic and mechanical transformation of formulas leading to inference. In traditional logic, deduction is carried out by invoking 10 rules of replacement together with some rules of inference; these rules announce that certain conclusions follow from certain sets of premises. Some logic-texts list hundreds of inference rules, while others make good efforts to summarize and classify them ${ }^{[1,2]}$ Table 1 includes a concise summary of inference rules. Each of the rules in Table 1 is famous enough to warrant having a name of its own.

In this research, we deal with a more general inference problem. We do not ask simply: Can a given proposition be inferred? but we ask What propositions relevant to a given question can be inferred? This more general problem is called a problem of logical projection $^{[3]}$ and is solved herein by a very powerful technique, which we call the Modern Syllogistic Method (MSM). An early but incomplete attempt to produce such a method appeared $\mathrm{in}^{[4]}$. The first popular correct description for the method is given by ${ }^{[5]}$. Other presentations of the method followed ${ }^{[6-9]}$. The great advantages of the method are that it ferrets out from a given set of premises all that can be concluded from it and that it casts these conclusions in the simplest or most compact form. The core step in the MSM is dual

Corresponding Author: Ali Muhammad Rushdi, Department of Electrical and Computer Engineering, King Abdulaziz University, P.O. Box 80204, Jeddah 21589, Saudi Arabia 
J. Math. \& Stat., 4 (3): 186-193, 2008

Table 1: Proof that each of the famous rules of inference is derivable by the MSM \{the particular conclusion of a rule is highlighted in bold\}

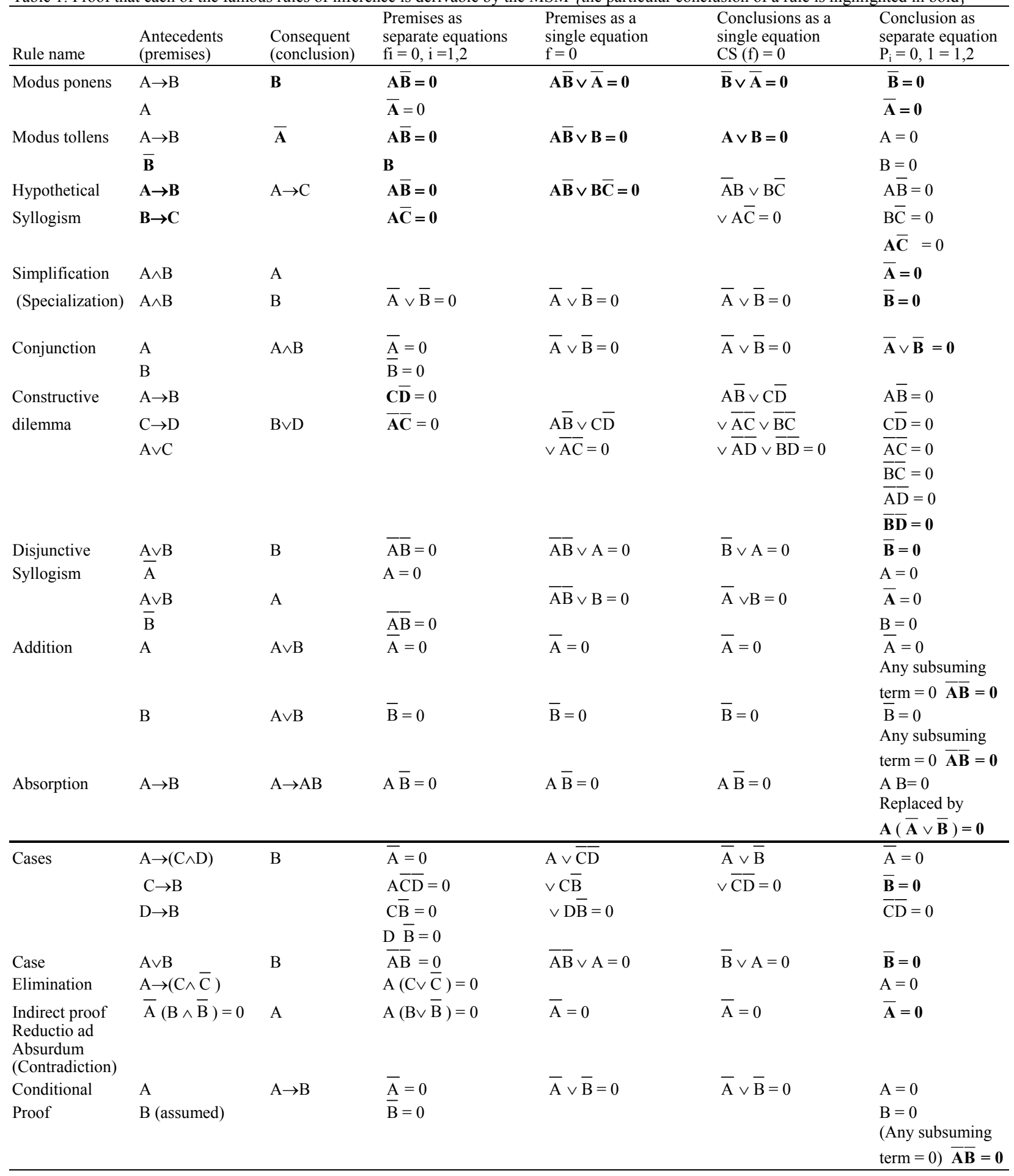

to the resolution principle in predicate logic ${ }^{[10,11,12]}$. This principle is used as a basis for automated reasoning employing non-procedural declarative logic programming languages such as $\operatorname{PROLOG}^{[13]}$.

\section{MATERIALS AND METHODS}

The methodology adopted herein is one of mathematical analysis, proof and demonstration by way 
of examples. In the following, we describe the MSM and explain the various techniques of switching algebra (two-valued Boolean algebra) needed for its implementation.

Description of the MSM: Information is conveyed in conventional real algebra by equations. Boole ${ }^{[14,15]}$ and other logicians of the past two centuries therefore found it natural to write logical statements as equations. Such equations are usually reduced to a single equivalent equation of the form:

$$
f(X)=0
$$

where, $\mathrm{f}$ is a switching or two-valued Boolean function while $X=\left[X_{1} X_{2} \ldots X_{n}\right]^{T}$ is an n-tuple of symbols which represent classes of objects or propositions. Brown ${ }^{[15-17]}$ and Wheeler ${ }^{[18]}$ point out the existence of an axiom peculiar to the calculus of propositions, which is called the principle of assertion and may be stated as:

$$
\left[\mathrm{X}_{\mathrm{i}}=1\right]=\mathrm{X}_{\mathrm{i}}
$$

Equation 2 states or asserts that: To say that a proposition $X_{i}$ is true is to state the proposition itself. It is therefore possible in the calculus of propositions to dispense entirely with equations. If $\mathrm{f}(\mathrm{X})$ is a propositional (i.e., two-valued) function, then Eq. 1 may be stated equivalently by the proposition.

$$
\bar{f}(X)
$$

Due to this principle of assertion, most contemporary logicians have abandoned equations in the formulation of propositional logic. The MSM, however, symbolizes a revival or renaissance, of the older or classical equation-based approach. Such an approach might use a function equated to 0 , as given in 1 , or equivalently, it may employ a function equated to 1 .

\section{Steps of the MSM:}

Step 1: Each of the premises is converted into the form of a formula equated to 0 (which we call equational form) and then the resulting equational forms are combined together into a single equation of the form $\mathrm{f}=0$. If we have $\mathrm{n}$ equivalence relations of the forms:

$$
\mathrm{T}_{\mathrm{i}}=\mathrm{Q}_{\mathrm{i}}, 1 \leq \mathrm{i} \leq \mathrm{n},
$$

they are set in the equational forms:

$$
\mathrm{T}_{\mathrm{i}} \overline{\mathrm{Q}}_{\mathrm{i}} \vee \overline{\mathrm{T}}_{\mathrm{i}} \mathrm{Q}_{\mathrm{i}}=0,1 \leq \mathrm{i} \leq \mathrm{n}
$$

We may also have (m-n) logical implication (logical inclusion) relations of the forms:

$$
\mathrm{T}_{\mathrm{i}} \rightarrow \mathrm{Q}_{\mathrm{i}},(\mathrm{n}+1) \leq \mathrm{i} \leq \mathrm{m}
$$

These relations symbolize the statements If $T_{i}$ then $\mathrm{Q}_{\mathrm{i}}$ or equivalently $\mathrm{T}_{\mathrm{i}}$ if only $\mathrm{Q}_{\mathrm{i}}$. Conditions (6) can be set into the equational forms:

$$
\mathrm{T}_{\mathrm{i}} \overline{\mathrm{Q}}_{\mathrm{i}}=0,(\mathrm{n}+1) \leq \mathrm{i} \leq \mathrm{m} .
$$

The totality of $\mathrm{m}$ premises in (4) and (6) finally reduce to the single equation $f=0^{[19]}$, where $f$ is given by:

$$
f=\bigvee_{i=1}^{n}\left(T_{i} \bar{Q}_{i} \vee \bar{T}_{i} Q_{i}\right) \vee \underset{i=(n+1)}{m} T_{i} \bar{Q}_{i}
$$

Equations 4 and 7 represent the dominant forms that premises can take. Other less important forms are discussed by ${ }^{[20]}$ and can be added to (8) when necessary.

Step 2: The function $\mathrm{f}$ in 8 is rewritten as a complete sum (Black canonical form), i.e., as a disjunction of all the prime implicants of $\mathrm{f}$. There are many manual and computer algorithms for developing the complete sum of a switching function $\mathrm{f}^{[5,21-23]}$. Most of these algorithms depend on two logical operations: (a) Consensus generation (or equivalently multiplying a product-of-sums into a sum-ofproducts) and (b) absorption.

Step 3: Suppose the complete sum of $\mathrm{f}$ takes the form:

$$
\mathrm{f}=\bigvee_{\mathrm{i}=1}^{\ell} \mathrm{P}_{\mathrm{i}}=0,
$$

where $P_{i}$ is the ith prime implicant of $f$. Equation 9 is equivalent to the set of equations:

$$
\mathrm{P}_{\mathrm{i}}=0,1 \leq \mathrm{i} \leq \ell .
$$

Equation 10 state in the simplest equational forms all that can be concluded from the original premises. The conclusions in (10) can also be cast into implication form. Suppose $\mathrm{P}_{\mathrm{i}}$ is given as a conjunction of uncomplemented literals $\mathrm{X}_{\mathrm{ij}}$ and complemented literals $\overline{\mathrm{Y}}_{\mathrm{ij}}$ i.e., 


$$
P_{i}=\bigwedge_{j=1}^{r} X_{i j} \wedge \bigwedge_{j=1}^{s} \bar{Y}_{i j}, 1 \leq i \leq \ell
$$

then, conditions (10) can be rewritten as:

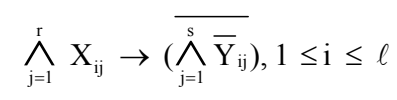

or as:

$$
\bigwedge_{\mathrm{j}=1}^{\mathrm{r}} \mathrm{X}_{\mathrm{ij}} \rightarrow \stackrel{\mathrm{s}}{\mathrm{s}=1}_{\mathrm{ij}}, 1 \leq \mathrm{i} \leq \ell
$$

We reiterate that the MSM produces all possible consequents (since CS (f) is a disjunction of all the prime implicants of $f$ and that it casts these consequents in the most compact form (since all the implicants in CS (f) are prime ones). If any implicant (whether it is prime or not) of $f$ is equated to 0 , then the result is a true consequent (albeit not necessarily in the most compact form). To test the truth of any claimed conclusion based on a given set of premises, one just needs to cast this conclusion in the form of a disjunction of terms equated to 0 and check to see if each of these terms subsumes (at least) one of the prime implicants in CS (f) derived for the set of premises.

\section{RESULTS AND DISCUSSION}

We show that the MSM is a complete method of logic deduction since it includes all rules (and hence all conventional methods) of propositional logic as special cases of it. Then we show that the MSM has a built-in capability of deducting the existence of inconsistency within a given set of premises and of demonstrating that inconsistent premises validly yield any conclusion whatsoever, no matter how irrelevant. We illustrate the use of the MSM to invalidate formal fallacies.

Completeness of the MSM: To demonstrate the power and completeness of the MSM, we introduce Table 1, which shows how this method can be used to derive each of the famous rules of inference. This amounts to a novel proof that each of these rules is a special case of the MSM. Note that the set of consequents of the MSM, being a complete set of conclusions, is usually a strict superset of the set of conclusions any rule of inference produces. The consequents of the MSM include all the premises of a certain rule, possibly simplified or rephrased, plus several new conclusions, of which only one is pointed out by the rule. For, example, the MSM can handle the three premises of the rule of constructive dilemma to produce six conclusions, of which three are simply echoes of the original premises and two are intermediate conclusions ignored by the rule, while the sixth is the ultimate conclusion of the rule. Table 1 is a major contribution of this paper, since it demonstrates definitely that the MSM encompasses a complete set of inference rules. Winnie ${ }^{[24]}$ and Copi ${ }^{[25]}$ showed that the list of 10 replacement rules together with the top 9 inference rules in Table 1 constitute a complete system of truth-functional logic, in the sense that it permits the construction of a formal proof of validity for any valid truth-functional argument. In fact these 19 rules are somewhat redundant, in the sense that they constitute more than a bare minimum which would suffice for the construction of formal proofs of validity for extended arguments ${ }^{[2]}$.

Handling inconsistencies: The MSM has a built-in capability of detecting the existence of inconsistency within a given set of premises and of explicitly demonstrating the ramifications of such inconsistency. If a set of premises is inconsistent, then their conjunction should be false, which means that the function $\mathrm{f}$ in their collective equational representation (1) should be equal to 1 . However, this fact is usually not obvious, but it can be brought to light easily through the MSM, which computes CS (f) naturally in its usual procedure for any set of premises and finds it to be equal to 1 if and only if such a set is inconsistent. The result:

$$
\mathrm{CS}(\mathrm{f})=1
$$

has two important aspects:

- Inconsistent premises (even when their inconsistency is highly concealed) lead to the selfevident contradiction $1=0$, indicating that the conjunction of the premises is self contradictory, i.e., no truth functional assignment can make all the premises true simultaneously

- Inconsistency of premises means that every consequent is true, since every term subsumes the term 1. Therefore, inconsistent premises can be used to assert the truth of any consequent to which the premises are totally irrelevant and to assert simultaneously the truth of any statement and its denial or contradictory statement

The above discussion shows that a user of the MSM is immune against falling into the trap of using a set of inconsistent premises to derive any conclusion. The method will alert its user to the concealed inconsistencies by producing CS (f) $=1$. Here the user 
should refrain from making any conclusion and should revise his set of premises to change it into a consistent one. The above discussion also demonstrates a possible way for a notorious misuse of logic. To prove any conclusion whatsoever, all one needs is to support it by a set of inconsistent premises, preferably (but not necessarily) with the inconsistency concealed as much as possible and with some apparent or fictitious relevance of the premises to the desired conclusion.

Invalidating formal fallacies: Historically, logic has been misused by being manipulated to give some sort of proof for false propositions. When one understands this, it is possible to identify the pitfalls (s) within the whole process, which are sometimes hidden in not-sothoroughly-investigated premises, but are occasionally due to the use of formal fallacies or incorrect rules of inference ${ }^{[26]}$. The MSM can be used in detecting and invalidating such formal fallacies. One of the inference rules in Table 1 is Modus Ponens, which asserts that premises $(\mathrm{A} \rightarrow \mathrm{B})$ and $\mathrm{A}$ lead to consequent $\mathrm{B}$. A similar purported rule claims that premises $(\mathrm{A} \rightarrow \mathrm{B})$ and $\mathrm{B}$ leads to consequent $\mathrm{A}$. For this purported rule, the MSM combines the premises in the single-equation form:

$$
f=A \bar{B} \vee \bar{B}=0
$$

from which one concludes that:

$$
\mathrm{CS}(\mathrm{f})=\overline{\mathrm{B}}=0
$$

Therefore, the claimed consequent $(\overline{\mathrm{A}}=0)$ is not asserted by the premises. This purported rule is an invalid argument and is called the converse fallacy ${ }^{[27]}$ or the fallacy of affirming the consequent ${ }^{[28]}$.

Another purported rule (whose shape is somewhat like that of Modus Tollens) claims that premises $(\overline{\mathrm{A}} \rightarrow \overline{\mathrm{B}})$ and A lead to consequent B. Again, the MSM combines the premises of this purported rule, to obtain the single equation:

$$
\mathrm{f}=\overline{\mathrm{A}} \mathrm{B} \vee \overline{\mathrm{A}}=0
$$

from which one obtains:

$$
\mathrm{CS}(\mathrm{f})=\overline{\mathrm{A}}=0
$$

The only consequent of the given premises is $(\overline{\mathrm{A}}=0)$ which is irrelevant to the claimed consequent $(\overline{\mathrm{B}}=0)$. This purported rule is again an invalid argument and is called the inverse fallacy ${ }^{[27]}$ or the fallacy of denying the antecedent ${ }^{[28]}$.
We present four examples to illustrate the mathematical details of the method, demonstrate its applicability in many diverse fields and exhibit the nature of conclusions it can come up with.

Example 1: What can be concluded from the following premises?

- If the mind and brain are identical, then the brain is a physical entity if and only if the mind is a physical entity

- If the mind is a physical entity then thoughts are material entities

- Thoughts are not material

- The brain is a physical entity

To rewrite these premises in symbolic form, we define the following pertinent binary variables:

$\mathrm{I}=$ The mind and brain are identical

$\mathrm{B}=$ The brain is a physical entity

$\mathrm{T}=$ Thoughts are material entities

$\mathrm{M}=$ The mind is a physical entity

Statements 1 through 4 above may be translated into symbolic form as follows:

$$
\begin{array}{ll}
\text { Clausal form } & \text { Equational form } \\
\mathrm{I} \rightarrow(\mathrm{B} \equiv \mathrm{M}) & \mathrm{I}(\mathrm{B} \overline{\mathrm{M}} \vee \overline{\mathrm{B}} \mathrm{M})=0 \\
\mathrm{M} \rightarrow \mathrm{T} & \mathrm{M} \overline{\mathrm{T}}=0 \\
\overline{\mathrm{T}} & \mathrm{T}=0 \\
\mathrm{~B} & \overline{\mathrm{B}}=0
\end{array}
$$

Then the given data are equivalent to the propositional equation $\mathrm{f}=0$, where $\mathrm{f}$ is given by:

$$
\mathrm{f}=\mathrm{IB} \overline{\mathrm{M}} \vee \mathrm{I} \overline{\mathrm{B}} \mathrm{M} \vee \mathrm{M} \overline{\mathrm{T}} \vee \mathrm{T} \vee \overline{\mathrm{B}} .
$$

The complete sum of $\mathrm{f}$ is:

$$
\mathrm{CS}(\mathrm{f})=\mathrm{I} \vee \mathrm{M} \vee \mathrm{T} \vee \overline{\mathrm{B}}=0 .
$$

The associated prime clauses are therefore the following:

$\mathrm{I}=0\{$ The mind and brain are not identical $\}$

$\mathrm{M}=0$ \{The mind is not a physical entity\}

$\mathrm{T}=0$ \{Same as premise 3$\}$

$\overline{\mathrm{B}}=0\{$ Same as premise 4$\}$

Is it possible to infer from the premises that $\{\mathrm{I} \rightarrow \mathrm{B}\}$ ? The answer is yes since the required 
conclusion is equivalent to the equation $\mathrm{I} \overline{\mathrm{B}}=0$ and the term I $\bar{B}$ subsumes the prime implicant I (or the prime implicant) $\bar{B}$ in (20). Is it possible to conclude from the premises that $\{\mathrm{B} \equiv \mathrm{M}\}$ ? The answer is no since the required conclusion is equivalent to the equation $B \bar{M} \vee \bar{B} M=0$ and the term $B \bar{M}$ therein does not subsume any of the prime implicants in (20).

Example 2: By the end of the eighteenth century, the common explanation of combustion was that something called phlogiston leaves a burning object, leaving only ashes behind. Let us consider the following premises:

- There is a combustible material to be burnt (B)

- If a material is burnt, the phlogiston within it is driven off into the air $(\mathrm{B} \rightarrow \overline{\mathrm{P}})$

- The phlogiston has a positive mass (M)

- Since the phlogiston has a positive mass, its loss by a certain material reduces the mass of that material $(\overline{\mathrm{P}} \mathrm{M} \rightarrow \mathrm{R})$

- A crucial experiment by Lavoisier in 1775 demonstrated that a burnt material undergoes an increase in mass $(\mathrm{B} \rightarrow \overline{\mathrm{R}})$

The following premises has the equational forms $\overline{\mathrm{B}}=0, \mathrm{BP}=0, \overline{\mathrm{M}}=0, \overline{\mathrm{P}} \mathrm{M} \overline{\mathrm{R}}=0$ and $\mathrm{BR}=0$. These can be combined into a single equation:

$$
f=\bar{B} \vee B P \vee \bar{M} \vee \bar{P} M \bar{R} \vee B R=0
$$

The function $f$ in (21) has a complete sum:

$$
\mathrm{CS}(\mathrm{f}) \equiv 1
$$

which, means that there is a contradiction $(1=0)$ indicating that the above set of premises is inconsistent. The inconsistency can be removed by changing at least one of the given premises, e.g., (a) assuming the phlogiston has a negative mass, or (b) abandoning the phlogiston theory altogether. In fact, when the phlogiston concept was deserted, the way was paved for the discovery of oxygen.

Example 3: By the end of the nineteenth century, the common explanation of the propagation of electromagnetic waves in vacuum is that vacuum is filled by a material medium called the ether. Let us consider the following premises:

- Time varying electromagnetic phenomena produce waves that are known to propagate in all types of $\operatorname{space}(\mathrm{E} \rightarrow \mathrm{W})$
- A wave must have a material medium for its propagation $(\mathrm{W} \rightarrow \mathrm{M})$

- The ether fills all space (including vacuum) so that space becomes filled with a material medium $(\mathrm{M} \rightarrow \mathrm{T})$

- There is light (L).

- Light is a form of electromagnetic waves $(\mathrm{L} \rightarrow \mathrm{E})$

- If ether exists, then the speed of light is different in the directions parallel and perpendicular to the direction of earth rotation $(\mathrm{LT} \rightarrow \mathrm{D})$

- In a crucial experiment by Michelson and Morley in 1887, there is no detectable difference in the speed of light in the aforementioned directions $(\mathrm{L} \rightarrow \mathrm{D})$

The foregoing premises has the equational forms $\mathrm{E} \overline{\mathrm{W}}=0, \quad \mathrm{~W} \overline{\mathrm{M}}=0, \quad \mathrm{M} \overline{\mathrm{T}}=0, \quad \overline{\mathrm{L}}=0, \quad \mathrm{~L} \overline{\mathrm{E}}=0$, $\mathrm{LT} \overline{\mathrm{D}}=0$ and $\mathrm{LD}=0$. These forms can be combined into the single equation:

$$
\begin{aligned}
& f=E \bar{W} \vee W \bar{M} \vee M \bar{T} \vee \bar{L} \\
& \vee L \bar{E} \vee L T \bar{D} \vee L D=0
\end{aligned}
$$

The function $\mathrm{f}$ in (23) has a complete sum:

$$
\mathrm{CS}(\mathrm{f}) \equiv 1
$$

which means that there is a contradiction $(1=0)$ and that there some inconsistency in the above set of premises or hypothesis. This set had to be revised. Important revisions are:

- The ether does not exist

- The electromagnetic wave can propagate in vacuum as well as in a material medium

- The speed of light in a specific medium is constant, irrespective of direction (and irrespective of observer location)

Example 4: The pigeonhole problem is to place $\mathrm{k}$ pigeons in $n$ holes so that no hole contains more than one pigeon. The problem is insoluble if $\mathrm{k}>\mathrm{n}$, for then some pigeonhole contains at least $\lceil\mathrm{k} / \mathrm{n}\rceil$ pigeons $^{[27]}$. Haken $^{[11]}$ and Chandru and Hooker ${ }^{[3]}$ showed that the pigeonhole problem gives rise to a logic formulation that is very hard to solve. Let us use $\mathrm{X}_{\mathrm{ij}}$ to denote that pigeon $\mathrm{i}$ is placed in hole $\mathrm{j}$. We need two types of premises:

- Premises to assert that each pigeon $i$ is placed in some hole 


$$
\bigwedge_{\mathrm{j}=1}^{\mathrm{n}} \overline{\mathrm{X}}_{\mathrm{ij}}=0,1 \leq \mathrm{i} \leq \mathrm{k}
$$

- Premises to assert, for each pair of pigeons, that both do not occupy the same hole

$$
\mathrm{X}_{\mathrm{ij}} \mathrm{X}_{\mathrm{lj}}=0,1 \leq \mathrm{j} \leq \mathrm{n}, 1 \leq \mathrm{i}<\mathrm{l} \leq \mathrm{k} .
$$

Now, consider the insoluble case of $\mathrm{k}=3$ and $\mathrm{n}=2$. Here, the conditions 25 and 26 combine to give:

$$
\begin{aligned}
& \mathrm{f}=\overline{\mathrm{X}}_{11} \overline{\mathrm{X}}_{12} \vee \bar{X}_{21} \overline{\mathrm{X}}_{22} \vee \overline{\mathrm{X}}_{31} \overline{\mathrm{X}}_{32} \\
& \vee \mathrm{X}_{11} \mathrm{X}_{21} \vee \mathrm{X}_{11} \mathrm{X}_{31} \vee \mathrm{X}_{21} \mathrm{X}_{31} \vee \\
& \mathrm{X}_{12} \mathrm{X}_{22} \vee \mathrm{X}_{12} \mathrm{X}_{32} \vee \mathrm{X}_{22} \mathrm{X}_{32}=0
\end{aligned}
$$

Finding the complete sum for the specific $f$ in (27) is very tedious compared with finding it for a typical 6variable function. It can be shown that:

$$
\mathrm{CS}(\mathrm{f})=1
$$

Equation 24 means that the set of premises 25 and 26 for the pigeonhole problem with $\mathrm{k}=3$ and $\mathrm{n}=2$ is inconsistent, there is no way to place 3 pigeons individually in 2 holes, since there is no way to make all the premises true simultaneously. Moreover, this set of inconsistent premises validly yields any conclusion, no matter how irrelevant. For example, consider the statements:

$\mathrm{E}=$ The earth is round

$\overline{\mathrm{E}}=$ The earth is not round

Since CS (f) $=1$ and the term 1 is subsumed by any term, including each of the terms $\bar{E}$ and $E$, then each of the results $(\bar{E}=0)$ and $(E=0)$ follows, leading to the simultaneous paradoxical assertion of the irrelevant statement $\mathrm{E}$ and its denial $\overline{\mathrm{E}}$.

A soluble (and simple) case of the pigeonhole problem is that of $\mathrm{k}=\mathrm{n}=2$ which has 2 distinct solutions. Here, the conditions 25 and 26 combine to give:

$$
\begin{aligned}
& \mathrm{f}=\overline{\mathrm{X}}_{11} \overline{\mathrm{X}}_{12} \vee \overline{\mathrm{X}}_{21} \overline{\mathrm{X}}_{22} \\
& \vee \mathrm{X}_{11} \mathrm{X}_{21} \vee \mathrm{X}_{12} \mathrm{X}_{22}=0
\end{aligned}
$$

Among 4-variable functions, the function $\mathrm{f}$ in 29 is usually cited as one whose $\mathrm{CS}$ is relatively hard to find $^{[21,23]}$. It can be shown ${ }^{[23]}$ that the expression 25 for $\mathrm{f}$ represents one minimal sum of $\mathrm{f}$ comprising 4 prime implicants, while CS (f) consists of 12 prime implicants. To solve the problem, however, we complement 29 and express $\overline{\mathrm{f}}$ in a disjoint sum-ofproducts form ${ }^{[19]}$.

$$
\begin{aligned}
& \bar{f}=X_{11} \bar{X} 12 \bar{X} 21 X_{22} \\
& \vee \bar{X} 11 X_{12} X_{21} \bar{X} 22=1
\end{aligned}
$$

This leads to the 2 solutions:

$$
\mathrm{X}_{11} \overline{\mathrm{X}}_{12} \overline{\mathrm{X}}_{21} \mathrm{X}_{22}=1,\left(\mathrm{X}_{11}=\overline{\mathrm{X}}_{12}=\overline{\mathrm{X}}_{21}=\mathrm{X}_{22}=1\right)
$$

which means placing pigeon 1 in hole 1 and pigeon 2 in hole 2 and:

$$
\overline{\mathrm{X}}_{11} \mathrm{X}_{12} \mathrm{X}_{21} \overline{\mathrm{X}}_{22}=1,\left(\overline{\mathrm{X}}_{11}=\mathrm{X}_{12}=\mathrm{X}_{21}=\overline{\mathrm{X}}_{22}=1\right)
$$

which means placing pigeon 1 in hole 2 and pigeon 2 in hole 1 .

This research describes the modern syllogistic method (MSM), which ferrets out from a given set of premises all the consequents that can be concluded from it and casts these consequents in the simplest compact form. The modern syllogistic method is similar to all other techniques of propositional logic in two respects: (a) it deals with arguments of many varieties on many topics including science, engineering, medicine, ethics, games and simple affairs of everyday life, (b) it concerns itself only with the form or quality of the arguments it handles and has nothing to do with their subject matter. The MSM distinguishes itself, however, among techniques of propositional logic, since it is the most powerful method among them and it encompasses each other technique as a special case. We believe that the MSM can serve as a useful tool for any researcher, as it can help him reason well about his discipline. A person mastering the method cannot be guaranteed to reason well or correctly, but he is more likely to reason correctly than one who is unaware of it. Partly this is because a person knowledgeable about the method can easily avoid the misuse of inconsistent premises to establish irrelevant conclusions and can also detect many kinds of common formal fallacies or errors in reasoning.

As a formal technique of logic, the MSM concerns itself only with the form of its premises and consequents and has nothing to do with their subject matter. It is up to the user of the method to use plausible heuristics to formulate the premises and interpret the consequents. The intervening task of going 
from the formal premises to the formal consequents is tackled in a completely algorithmic fashion by the method. By contrast, heuristics required of the user are fallible, involve some linguistic and verbal elements and cannot be replaced by exact or recipes of algorithms.

\section{REFERENCES}

1. Klenk, V., 2007. Understanding Symbolic Logic. 5th Edn., Prentice-Hall, Englewood Cliffs, NJ.

2. Copi, I.M. and C. Cohen, 2002. Introduction to Logic. 11th Edn., Prentice-Hall, Upper Saddle River, NJ.

3. Chandru, V. and J.N. Hooker, 1999. Optimization Methods for Logical Inference. 1st Edn., Wiley, New York, NY.

4. Lynch, E.P., 1980. Applied Symbolic Logic. Ist Edn., Wiley, New York, NY.

5. Brown, F.M., 1990. Boolean Reasoning: The Logic of Boolean Equations. 2nd Edn., Kluwer Academic Publishers, Boston, MA. Dover Publications, Mineola, NY.

6. Gregg, J.R., 1998. Ones and Zeroes: Digital Circuits and the Logic of Sets. 1st Edn., Wiley, New York, NY.

7. Rushdi, A.M. and A.S. Al-Shehri, 2002. Logical reasoning and its role in serving security and justice. Sec. Res. J., 11: 114-153 (In Arabic with an English abstract).

8. Rushdi, A.M. and O.M. Ba-Rukab, 2007. Some engineering applications of the modern syllogistic method. Proceedings of the $7^{\text {th }}$ Saudi Engineering Conference: Riyadh, 2-5 December 2007, King Saud University Press, 4: 389-401.

9. Rushdi, A.M. and O.M. Ba-Rukab, 2008. The modern syllogistic method as a tool for engineering problem solving. J. Qassim University: Eng. Comput. Sci., 1: 1-22.

10. Robinson, J.A., 1965. A machine oriented logic based on the resolution principle. J. Associat. Comput. Mach., 12: 23-41.

11. Haken, A., 1985. The intractability of resolution. Theoretical Comput. Sci., 39: 297-308.

12. Chang, Chin-Liang and R.C.T. Lee, 1997. Symbolic Logic and Mechanical Theorem Proving. 2nd Edn., Academic Press, New York.
13. Russel, S. and P. Norvig, 2002. Artificial Intelligence: A modern Approach. 2nd Edn., Prentice-Hall, Englewood Cliffs, NJ.

14. Boole, G., 1847. The Mathematical Analysis of Logic. G. Bell, London. (Reprinted by Thoemmes Press, 1998).

15. Boole, G. 1854. An Investigation of the Laws of Thought. Walton, London. (Preprinted by Dover Publications, Mineola, NY, 1954).

16. Brown, F.M. 1974. Equational logic. IEEE Trans. Comput., 23: 1228-1237.

17. Brown, F.M., 1975. Equational realizations of switching functions. IEEE Trans. Comput., 24: 1054-1066.

18. Wheeler, R.F., 1981. Rethinking Mathematical Concepts. 1st Edn., Prentice-Hall, Englewood Cliffs, NJ.

19. Rushdi, A.M., 2001. Using variable-entered Karnaugh maps to solve Boolean equations. Int. J. Comput. Math., 78: 23-38.

20. Klir, G.J and M.A. Marin, 1969. New considerations in teaching switching theory. IEEE Trans. Educ., 12: 257-261.

21. Muroga, S., 1979. Logic Design and Switching Theory. 1st Edn., Wiley, New York, NY.

22. Rushdi, A.M., 2001. Prime-implicant extraction with the aid of the variable-entered Karnaugh map. Umm Al-Qura University J. Sci., Med. Eng., 13: 53-74.

24. Rushdi, A.M. and H.A. Al-Yahya, 2001. Derivation of the complete sum of a switching function with the aid of the variable-entered Karnaugh map. King Saud University J. Eng. Sci., 13: 239-269.

25. Winnie, J.A., 1970. The completeness of Copi's system of natural deduction. Notre Dame J. Formal Logic., 11: 379-382.

26. Copi, I.M., 1979. Symbolic Logic. 5th Edn., Prentice hall, New York, NY.

27. Nelson, I.T., R.L. Ratliff, G. Steinhoff and G.J. Mitchell, 2003. Teaching logic to auditing students: can training in logic reduce audit judgment errors. J. Account. Educ., 21: 215-237.

28. Anderson, J.A., 2003. Discrete Mathematics with Combinatorics. 1st Edn., Prentice Hall, Upper Saddle River, NJ. 